\title{
Appraisal of Recognition Methods
}

\author{
Sina.Layazali \\ Department of Computer Eng \\ Çankaya University, Ankara, Turkey
}

\author{
Yaser Ahangari N. \\ Department of IT \\ Çankaya University, Ankara, Turkey
}

\begin{abstract}
Today many systems are invented which have special ability that sense of recognition plays a vital role in them and reshaped our life dramatically. These kinds of systems can make a proper decision about data clustering. Image processing or recognition of patterns like signs and ... are the systems that are the example of them. Till now many methods have been presented which used to design a recognition system that some of them haven't been improved completely. Nevertheless, most of them should follow some steps such as: how to identify and represent the classes, the manner of choosing and extracting and how to cluster and train samples. Although, some issues like orientation or location couldn't be solved by these systems but scholars have trying to find the best solution for them. In this article, primary goal is comparing the methods used in pattern recognition that neural network is one of the most important techniques among them.
\end{abstract}

\section{Keywords}

Statistical pattern recognition, classification, clustering, feature extraction, feature selection, error estimation, neural networks.

\section{INTRODUCTION}

In fact pattern recognition is the study about the methods is used by machines to make a decision in different fields. Overall, fields of patterns have been divided into supervised and unsupervised [14]. As far as it has been mentioned the goal of this article is to represent of methods like template matching, structural matching, neural network and statistical classification used to design a recognition system which follows three steps called: The design of pattern recognition system essentially involves the following three aspects: How to acquire the data and preprocessing, Data representation and make a suitable decision [2][15].

\subsection{Concept of pattern recognition}

To define the meaning of the classification of patterns briefly and simply, it can be said that it is oppose of chaos [10]. Today in some fields, requirement of some automatic process such as classification or description or even make group of some entities which have a together, have become an urgent need. Overall, classification of patterns should follow neither of two methods based on supervised and unsupervised. In supervised classification method, patterns are belonged to specific classes which are defined before. On the other hand, classes which patterns are going to belong haven't identified. In fact pattern recognition act like human's brain to make a good decision. Many computers are used to decide in different field like face and fingerprint processes and so on. However, computers have problem about getting decision in emotion filed. [1] [30]

\subsection{Template Matching}

One of the simplest and earliest approaches to pattern recognition is based on template matching which is used to determine the similarity between two entities [3]. One kind of template or one entity of a pattern is compared with a pattern stored. Also, common factors based on the available training set will be optimized and templates teach their self from training set.

\subsection{Statistical Approach}

In this approach patterns presented in terms of modules which is viewed as a point in a d-dimensional space. $\mathrm{d}$-dimensional space is a form of perimeter where pattern vectors from different groups try to get permission to hold regions by features. As it is obvious each pattern is member of each class and in this approach, by computing the probability distributions of the patterns, system can distinguish the edge of decision. In addition, decision boundary can be thought or be defined. The best method to make a decision boundary is based on classification of training patterns [4] [5].

\subsection{Syntactic Approach}

Sometimes recognition systems face with some problems such as complex patterns [6]. This method acts like a divider of a structure of a language that principles are the alphabet and patterns play their roles as long sentences of language. So, by Syntactic approach can divide the complex patterns to the elementary parts that is called primitive. In fact this manner illustrates the construction of each pattern than classification [7].Fu has presented a theory that incorporates the syntactic and statistical of pattern recognition. Syntactic Approach can result the combination of large possibilities that stem from training sets and many number of computational endeavor [8].

\subsection{Neural Networks}

To begin, the task of neural network is to classify the patterns are feed-forward and self-organizing map [9]. Based on Neural network rules, in this approach nodes and edges are the artificial and the bonds which are between input and output of neurons. It is interesting to say that Ripley[11] [12] has focused on relationship between neural and statistical recognition. Neural networks have special ability like cognition of relevance between twisted nonlinear inputoutput, adjust themselves to data and use consecutive training procedures. In addition there are some advantages of neural network for classification of patterns such as having federation attitude about extraction and classification of features and using methods that their task is, finding the proper solution pliability [13]. 


\section{Statistical of Pattern Recognition}

As far I mentioned, in statistical pattern recognition patterns are represented by a set of $\mathrm{d}$ - features and viewed as $\mathrm{d}$ dimensional feature vector. The recognition system is operated in two modes called training and testing that there is a common step called preprocessing which its duty is to emit noises, fragment the pattern of interest from the background, and any other operation which will contribute in defining a compact representation of the pattern. In training method feature extraction/selection finds the appropriate features for representing the input patterns and in second method, Classifier is trained to partition the feature space.
Furthermore there are other ideas which are opposite like supervised learning and unsupervised learning or Geometric approach and Probabilistic density-based approach. Based on references geometric approach often constructs the decision boundaries directly from the optimizing certain cost functions and Probabilistic density-based approach estimate density functions and then discriminant functions. In figure number2 some dichotomies are presented.

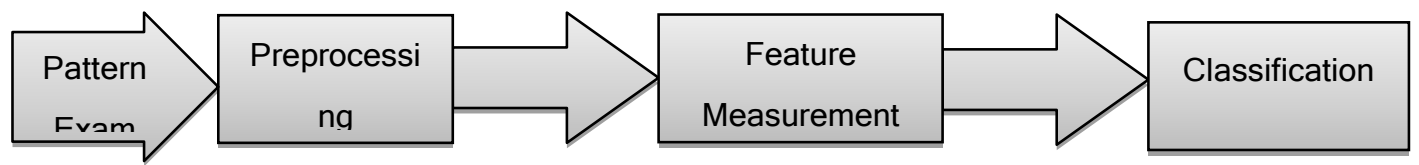

Classification

\section{Training}

Figure.1

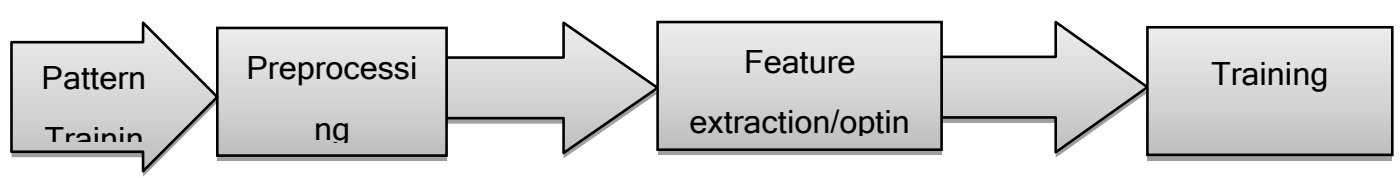

The feedback path allows a designer to optimize the preprocessing and feature selection/extraction strategies. To define a decision boundary some important principles methods listed Bayes decision, Maximum likelihood rule (particular case of Bayes decision), and Neyman-Pearson. Bayes decision for underrating the risk follows:

$$
\begin{gathered}
R\left(\omega_{\mathrm{i}} \mid \mathrm{x}\right)=\sum_{\mathrm{j}=1}^{\mathrm{c}} \mathrm{L}\left(\omega_{\mathrm{i}}, \omega_{\mathrm{j}}\right) \cdot \mathrm{P}\left(\left(\omega_{\mathrm{i}} \mid \mathrm{x}\right)\right. \\
\mathrm{L}\left(\omega_{\mathrm{i}}, \omega_{\mathrm{j}}\right)= \begin{cases}0, & \mathrm{i}=\mathrm{j} \\
1, & \mathrm{i} \neq \mathrm{j}\end{cases} \\
\mathrm{P}\left(\left(\omega_{\mathrm{i}} \mid \mathrm{x}\right)>P\left(\left(\omega_{\mathrm{i}} \mid \mathrm{x}\right) \text { for all } \mathrm{j} \neq \mathrm{i}\right.\right.
\end{gathered}
$$

Depending on the given information about class-conditional densities, many different strategies are used to design a classifier [15]. If all of the class-conditional densities are completely specified, it will be Optimal Bayes decision rule. Some of the parametric of the densities are unknown. So, computing parametric boundary will be an extremely problem. This kind of parametric is called Bayes plug -in classifier. For this problem one solution has been presented which is to replace the unknown parameters in the density functions by their estimate values. In addition, optimal strategy asks more information in the form of a prior distribution on the unknown parameters.In another situation called nonparametric mode if the class-conditional densities are not known. Estimation of the density function or construct the decision boundary based on training data will be needed.
Opposite of how to make decision about which decision rule is proper that is not important; it must trained using available training samples. The performance of each classifier which is used to classify the patterns were not used during the training stage shows the ability of the classifier. There are two important factors that lead the classifier to have poor generalization ability. Firstly, it is related to the features that are much larger than training samples. Secondly, a classifier is optimized intensively. Finally, the quantity of unknown parameters related to classifier is large [22][23] [24]. All over the paper some methods related to classification follow three important data set: 1.An artificial dataset which include two classes with bivariate Gaussian density. 2. Iris dataset consists three classes that have 50patterns (iris versicolor, iris setosa, iris virginica). 3. The digit data comprises handwriting numbers.

\section{Conclusion}

In this article some trends and recognition methods with advantages and drawbacks have been introduced. In addition, among second principal step called statistical of pattern recognition, some strategies based on different situation for design a recognition system have been presented. Testing and training are the most important points of that stage. During last decade in field of recognition, many applications like face recognition has been presented that can do their tasks successfully. These applications don't need variety of features. However; there are some applications do not need invariant features. Template matching and neighbor classifier which are mentioned about them, are the methods used in second group of application. One of the last recognition applications is hypertext classifier that can be improved in following years. Since many years ago many articles have been released that introduced many methods with different 
topics like curse of dimensionality, dimensionalityreduction, classifier design, classifiercombination, error estimation and unsupervised classification [14][15][16][17][18][19] [20][21].

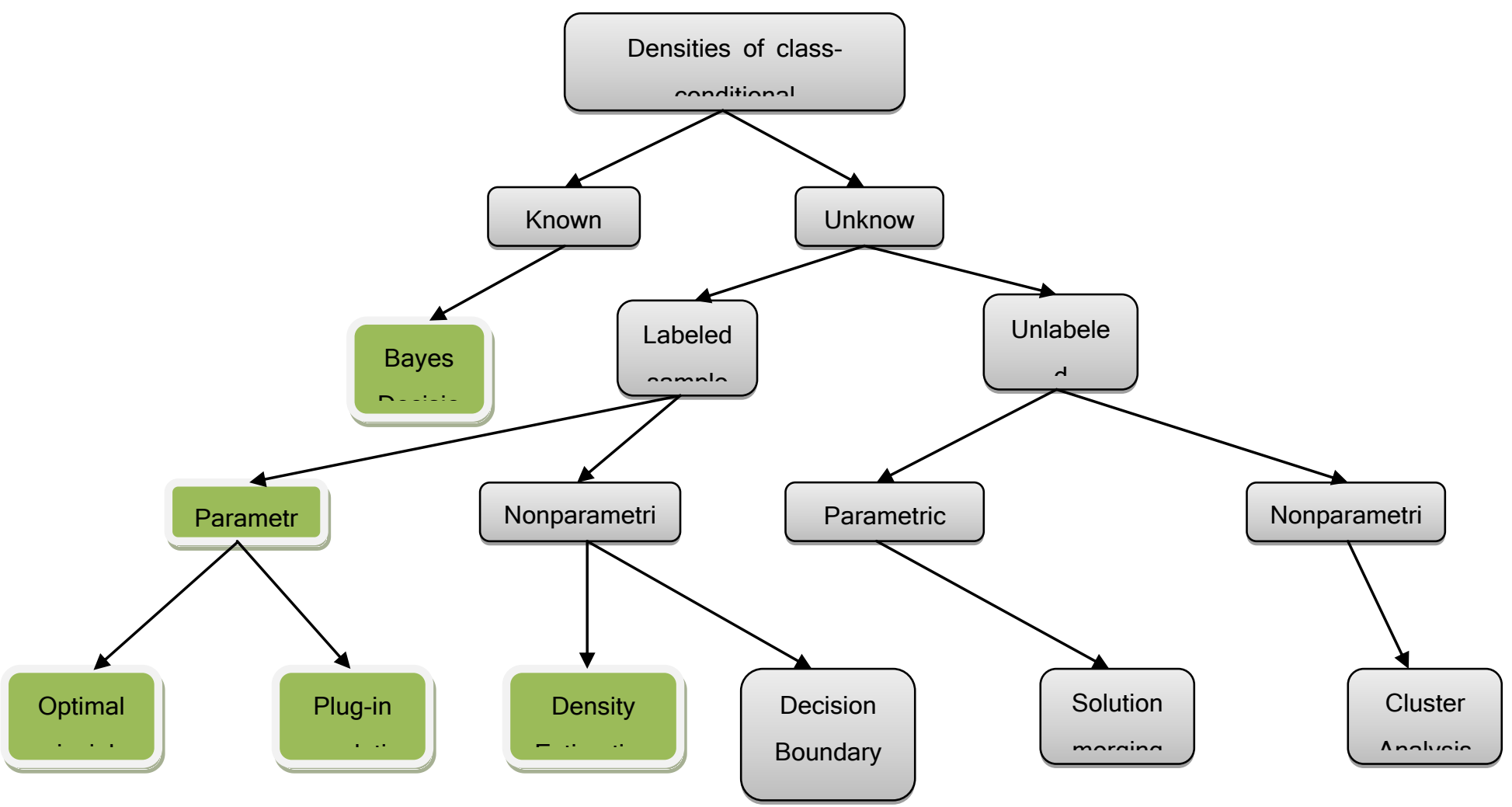

Figure.2

\section{References}

[1] R. Picard, Affective Computing.MIT Press, 1997.

[2] K.S. Fu, aA Step Towards Unification of Syntactic and StatisticalPattern Recognition, ${ }^{\circ}$ IEEE Trans. Pattern Analysis and Machine Intelligence, vol. 5, no. 2, pp. 200205, Mar. 1983.

[3] U. Grenander, General Pattern Theory. Oxford Univ.Press, 1993.

[4] L. Devroye, L. Gyorfi, and G. Lugosi, A Probabilistic Theory of Pattern Recognition. Berlin: Springer-Verlag, 1996.

[5] R.O. Duda and P.E. Hart, Pattern Classification and Scene Analysis, New York: John Wiley \& Sons, 1973.

[6] K.S. Fu, Syntactic Pattern Recognition and Applications.Englewood Cliffs, N.J.: Prentice-Hall, 1982.

[7] T. Pavlidis, Structural Pattern Recognition. New York: Springer- Verlag, 1977.

[8] L.I. Perlovsky, 'Conundrum of Combinatorial Complexity,' IEEE Trans. Pattern Analysis and Machine Intelligence, vol. 20, no. 6, pp. 666-670, 1998.

[9] T. Kohonen, Self-Organizing Maps. Springer Series in InformationSciences, vol. 30, Berlin, 1995. J.Jensen, and W. Kendal, eds., Chapman and Hall, 1993.
[10] S. Watanabe, Pattern Recognition: Human and Mechanical. New York: Wiley, 1985.

[30] P.E. Ross, a Flash of Genius, ${ }^{\circ}$ Forbes, pp. 98-104, Nov. 1998.

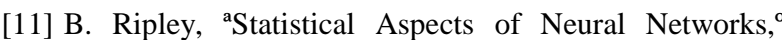
Networks on Chaos: Statistical and Probabilistic Aspects. U. Bornndorff-Nielsen, J. Jensen, and W. Kendal, eds. Chapman and Hall, 1993.

[12] J. Anderson, A. Pellionisz, and E. Rosenfeld, Neurocomputing 2: Directions for Research. Cambridge Mass.: MIT Press, 1990.

[13] J.C. Bezdek, Pattern Recognition with Fuzzy Objective Function Algorithms. New York: Plenum Press, 1981.

[14] F. Ferri, P. Pudil, M. Hatef, and J. Kittler, ${ }^{\text {aCComparative }}$ Study of Techniques for Large Scale Feature Selection, ${ }^{\circ}$ Pattern Recognition in Practice IV, E. Gelsema and L. Kanal, eds., pp. 403-413, 1994.

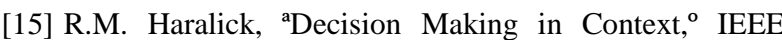
Trans. Pattern Analysis and Machine Intelligence, vol. 5, no. 4, pp. 417-418, Mar. 1983. Pattern Analysis and Machine Intelligence, vol. 5, no. 4, pp. 417- 418, Mar. 1983.

[16] C. Carpineto and G. Romano, ${ }^{\mathrm{a}} \mathrm{A}$ Lattice Conceptual Clustering System and Its Application to Browsing Retrieval, ${ }^{\circ}$ Machine Learning, vol. 24, no. 2, pp. 95-122, 
1996. System and Its Application to Browsing Retrieval, ${ }^{\circ}$ Machine Learning, vol. 24, no. 2, pp. 95-122, 1996.

[17] A. Barron, J. Rissanen, and B. Yu, ${ }^{a}$ The Minimum Description Length Principle in Coding and Modeling, ${ }^{\circ}$ IEEE Trans. Information Theory, vol. 44, no. 6, pp. 2,743-2,760, Oct. 1998 .

[18] K. Fukunaga, Introduction to Statistical Pattern Recognition. seconded., New York: Academic Press, 1990.

[18] C.M. Bishop, Neural Networks for Pattern Recognition. Oxford: Clarendon Press, 1995.

[19] J. Schurmann, Pattern Classification: A Unified View of Statistical and Neural Approaches. New York: John Wiley \& Sons, 1996.
[11] G. McLachlan, Discriminant Analysis and Statistical Pattern Recognition. New York: John Wiley \& Sons, 1992.

[21] L.N. Kanal, a'Patterns in Pattern Recognition: 1968-1974, ${ }^{\circ}$ IEEE Trans. Information Theory, vol. 20, no. 6, pp. 697 $722,1974$.

[22] A.K. Jain and R.C. Dubes, Algorithms for Clustering Data. Englewood Cliffs, N.J.: Prentice Hall, 1988.

[23] A.K. Jain and B. Chandrasekaran, a Dimensionality and Sample Size Considerations in Pattern Recognition Practice, ${ }^{\circ}$ Handbook of Statistics. P.R. Krishnaiah and L.N. Kanal, eds., vol. 2, pp. 835-855, Amsterdam: NorthHolland, 1982.

[24] L. Devroye, a Automatic Pattern Recognition: A Study of theProbability of Error, ${ }^{\circ}$ IEEE Trans. Pattern Analysis and Machine Intelligence, vol. 10, no. 4, pp. 530-543, 1988. 